\title{
Qualität gesundheitsbezogener Online-Angebote aus Sicht von Usern und Experten
}

\author{
Sabine Trepte / Eva Baumann / Nina Hautzinger / Gabriele Siegert
}

In der Gesundheitskommunikation wird dem Internet eine zunebmend wichtige Rolle zugeschrieben. Dabei ist aufgrund der Vielfalt an verfügbaren Inhalten, mangelnder Qualitätsstandards und der geringen formalen Expertise der User die Qualitätsbeurteilung von zentraler Bedeutung. Im Rabmen des Artikels werden zunächst Qualitätsdimensionen aus vorhergehenden Studien aggregiert und daraufhin die Bewertung und Vorbersagekraft der drei ausgewäblten Kriterien Inhaltsqualität, Darstellungsqualität und technische Qualität im Rabmen zweier Studien analysiert. Dazu wurden 101 User im Labor zu drei deutschsprachigen kommerziellen Gesundheitsportalen befragt. Es zeigt sich, dass User ibren ersten Eindruck einer Webseite nach einer gezielten Informationssuche (zu den Krankheiten Bandscheibenvorfall, Depression, Diabetes) spezifizieren. Als wichtige Prädiktoren für die Gesamtbewertung und Weiternutzung einer Webseite können die korrekte Darstellung der Krankheitsentstehung, die Vollständigkeit der dargestellten Inhalte und die übersichtliche Navigation auf der Webseite gelten. Die ergänzende Studie, in der sieben Experten zur Inhaltsqualität der Webseiten befragt wurden, stellte eine große Übereinstimmung von User- und Expertenurteilen heraus.

Stichwörter: Gesundheitskommunikation, Health Content, Internet, Qualität, Laborbefragung, Expertenbefragung, Inhaltsqualität, Darstellungsqualität, technische Qualität

\section{Einleitung}

In der Gesundheitskommunikation spielt das Internet eine zunehmend wichtige Rolle und eröffnet neue Perspektiven für die Gesundheitsförderung. Es bieten sich zahlreiche Möglichkeiten, den hohen Informations- und Kommunikationsbedarf von Experten, Patienten und Interessierten zu decken. Das Angebot an gesundheitsspezifischen Informationen im Internet lag bereits 1999 bei über 100000 geschätzten Webseiten und nimmt stetig zu (Manfredi \& Covington, 2000). Dieses Angebot trifft auf eine entsprechende Resonanz auf Rezipientenseite. Gesundheitsbezogene Themen gehören zu den am häufigsten abgerufenen Themen im Internet (Manfredi \& Covington, 2000), und immerhin 25 Prozent aller Personen, die in Deutschland 2003 einen Arzt aufgesucht haben, konsultierten vorher das Internet, um sich zu informieren (Emnid, 2003). „With the proliferation of health-related Websites, [...] the internet has unfolded as a major source of reliable information, education, and management processes about a variety of medical conditions and their treatment." (Manfredi \& Covington, 2000: 242)

Über das Internet können Gesundheitsinformationen einer breiten Masse von Menschen, die einen Internetzugang haben, zur Verfügung gestellt werden. Aber auch kleine, spezielle und weit verstreute Zielgruppen (z. B. an seltenen Krankheiten leidende Personen) lassen sich gut erreichen und in Kontakt zueinander bringen, sofern sie zum Kreis der Internetnutzer gehören (Neuhauser \& Kreps, 2003b). Durch die zahlreichen Anbieter gesundheitsbezogener Informationen wie Selbsthilfegruppen, Patientenorganisationen, staatliche Stellen, Pharmaunternehmen, kommerzielle Anbieter, Krankenkassen, Verlage, Stiftungen oder Privatpersonen hat sich das Informations- und Mei- 
nungsspektrum für Informationssuchende erweitert, und die bisherige Abhängigkeit von Ärzten und Apothekern verringert sich. Eine breite Informationsbasis kann helfen, Unsicherheiten abzubauen und die für die Inanspruchnahme gesundheitsrelevanter Dienstleistungen nötigen Entscheidungskompetenzen zu verbessern (Schmidt-Kaehler, 2003).

Gesundheitskommunikation im Internet birgt jedoch auch Gefahren, denn hochund minderwertige Informationen stehen kommentarlos nebeneinander, und ihre Qualität ist für Laien oft kaum zu durchschauen. Auf vielen Webseiten fehlen wichtige Kontextinformationen wie die Urheberschaft und die von den Anbietern verfolgten Interessen (vgl. Eysenbach et al., 2002). So können falsche Informationen oder der unangemessene Umgang mit Hinweisen für die User negative gesundheitliche Folgen haben (Schmidt-Kaehler, 2003).

Das vorhandene Potenzial des Internets für die Gesundheitskommunikation kann also nur dann ausgeschöpft werden, wenn User in der Lage sind, die Qualität der Online-Angebote angemessen einzuschätzen. Vor dem Hintergrund der zunehmenden Frequentierung von Gesundheitswebseiten und der großen inhaltlichen Relevanz gesundheitsbezogener Informationen für die User soll im Rahmen dieses Artikels die Frage beantwortet werden, wie User die Qualität von deutschsprachigen Gesundheitsportalen bewerten. Es soll gezeigt werden, welche Qualitätskriterien relevant für die Bewertung einer Webseite sind und von welchen Qualitätseinschätzungen es abhängt, dass eine Seite weiterhin genutzt wird. Um zu überprüfen, inwiefern es den Laien gelingt, qualitativ hoch- und minderwertige Inhalte zu unterscheiden, wird den Userurteilen eine Experteneinschätzung der Qualität der Webseiten gegenübergestellt.

Diese Fragestellungen werden für drei kommerzielle Webseiten (Lifeline.de, Gesmed.ch und Netdoktor.de) untersucht, in denen primär von Medizinern und Journalisten formulierte Texte zu unterschiedlichen Gesundheitsthemen und Service-Informationen aus unterschiedlichen Bereichen des Gesundheitswesens neben Werbebannern bereitgestellt werden. Es ist anzunehmen, dass bei Verflechtungen journalistischer Inhalte mit kommerziellen Angeboten Qualitätsurteile besonders schwer zu fällen sind (Neuberger, 2004). Urheberschaften und die Intentionen der Informationsanbieter sind schwerer zu durchschauen. Die Informationsqualität ist ein der Vermarktung möglicherweise untergeordnetes Ziel (vgl. Reiß, 2000). Die Frage nach der Qualitätsbeurteilung ist bei diesen Angeboten daher besonders brisant.

Im Folgenden werden zunächst die Nutzer und die Nutzung gesundheitsbezogener Informationen im Internet skizziert und die Handlungsrelevanz der Informationen aufgezeigt (Abschnitt 2). Anschließend wird der Qualitätsbegriff näher bestimmt (Abschnitt 3.1 bis 3.4). Auf Basis dieser Vorüberlegungen wird ein empirisches Forschungsdesign entwickelt, das eine User- und Expertenbefragung einschließt (Abschnitt 5), um die skizzierten Forschungsfragen (Abschnitt 4) zu beantworten (Abschnitt 6) und zu diskutieren (Abschnitt 7).

\section{Nutzer, Nutzung und Handlungsrelevanz von Gesundheitsinformationen im Internet}

Gesundheit wird den Deutschen immer wichtiger (Identity Foundation, 2001). Art und Ausmaß der Suche nach Gesundheitsinformationen im Internet hängen eng mit dem aktuellen Gesundheitsstatus einer Person zusammen. Kranke haben im Vergleich zu denjenigen, die sich selbst einen guten Gesundheitszustand attestieren, ein höheres themenspezifisches Involvement und suchen - häufig auf Anregung von Experten - intensiver nach Informationen über ihre Erkrankung (Bowen et al., 2003). Auch nutzen sie 
häufiger Chatforen (Houston \& Allison, 2002). Bei der Recherche zu Gesundheitsinformationen im Internet werden Erkrankte und Betroffene sehr häufig von ihren Angehörigen und Freunden unterstützt (Fox \& Fallows, 2003).

Welche Gesundheitsinformationen werden im Internet nun konkret gesucht und welchen Zweck sollen sie für die Nutzer erfüllen? Mehr als drei Viertel derjenigen, die Gesundheitsinformationen im Internet suchen, tun dies, wenn sie Informationen zu einem bestimmten Thema benötigen (Harris Interactive, 2001). Eine Befragung unter 1500 Usern auf dem Gesundheitsportal Lifeline.de ergab, dass es den Nutzern darauf ankommt, Informationen schnell einholen zu können, diese von kompetenten Experten zu erhalten und Hintergrundinformationen über aktuelle Gesundheitsfragen zu bekommen (BSMO, 2003). Fox et al. (2000) kommen zu dem Schluss, dass User eher Informationen aus dem Internet ziehen möchten als auf diesem Wege mit Experten oder Betroffenen in Kontakt zu treten oder andere Dienstleistungen in Anspruch zu nehmen. Gesucht werde dabei eher nach Krankheitsthemen als nach Themen zu Fitness und zur Aufrechterhaltung der Gesundheit.

Gemäß der typischen Internetnutzerstruktur sind diejenigen, die Gesundheitsinformationen im Internet suchen, eher jünger ( $81 \%$ unter 46 Jahre, $M=38$ Jahre) sowie eher gut gebildet und einkommensstark (BSMO, 2003). Neben dem Arztbesuch und der Lektüre von Fachzeitschriften sowie dem Gespräch mit Freunden und Bekannten stellt unter Internetnutzern das Internet die wichtigste Infoquelle dar (T-Online, 2002). Frauen kommt in Gesundheitsfragen eine Schlüsselrolle zu. Eher als Männer sammeln sie Informationen und Erfahrungen rund um das Thema Gesundheit, entscheiden in der Familie, ob ein Arztbesuch notwendig ist oder ob Medikamente sinnvoll sind. Dementsprechend sind 74 Prozent der gesundheitsinteressierten Internetnutzer weiblich (T-Online, 2002).

Insgesamt wird dem Internet in der Gesundheitskommunikation ein hohes, positives Wirkungspotenzial und praktischer Nutzen für das eigene Handeln zugeschrieben (z. B. Neuhauser \& Kreps, 2003a). Die meisten User von Gesundheitsinformationen im Netz sind mit dem inhaltlichen Angebot zufrieden. Laut Horrigan und Rainie (2002) findet die Mehrheit derjenigen, die Gesundheitsinformationen im Internet suchen, diese auch nützlich und berichtet, etwas Neues gelernt zu haben und den Informationen $\mathrm{zu}$ vertrauen (vgl. Houston \& Allison, 2002). Aus ihrer Sicht liegen die Vorteile des Internets als Quelle gesundheitsbezogener Informationen neben der Informationsvielfalt, der hohen Aktualität, dem relativ kostengünstigen, zeitflexiblen und bequemen Zugriff von Zuhause in der Möglichkeit, anonym zu bleiben und sich dennoch mit anderen auszutauschen. Dies kann gerade im Falle tabuisierter Erkrankungen ausschlaggebend sein.

Besorgt sind die User - und hier eher Frauen als Männer sowie insbesondere die weniger Internet-Erfahrenen - um den Schutz ihrer personenbezogenen Daten (Fox et al., 2000). Eine weitere Befürchtung der Nutzer bezieht sich auf die Glaubwürdigkeit der Informationen und Empfehlungen im Netz.

Die im Internet generierten Informationen können in verschiedener Weise und zu unterschiedlichen Kommunikationsanlässen handlungsrelevant werden. Etwa die Hälfte der Nutzer berichtet, dass der Ratschlag, den sie im Internet gefunden haben, unmittelbar dazu beigetragen hat, besser auf sich selbst zu achten, dass sie ihre Entscheidungen der eigenen Gesundheitsversorgung hieran orientiert und sogar ihr tatsächliches Gesundheitsverhalten verändert haben (Fox et al., 2000). Um ihr Informationsdefizit gegenüber Experten möglichst gut ausgleichen zu können und ihre Kompetenz in ihrer eigenen Gesundheitsversorgung zu verbessern, suchen Internetnutzer häufig im Vorfeld oder nach einer ärztlichen Diagnose im Netz Informationen zu Krankheiten oder zu ge- 
eigneten medizinischen Einrichtungen und bringen diese auch in das Arztgespräch ein (vgl. auch Houston \& Allison, 2002; Emnid, 2003). Der Arztbesuch wird hierdurch oft als konstruktiver und die Kommunikation mit dem Arzt als besser erlebt (Health On the Net Foundation, 2002). Auch bezogen auf deutschsprachige Angebote bescheinigen die Teilnehmer einer von Eysenbach und Köhler (2002) durchgeführten qualitativen Mehrmethodenstudie den gesundheitsbezogenen Webseiten eine hohe Qualität. Die 21 Probanden begrüßten die Möglichkeiten, durch das Internet Aussagen von Ärzten zu überprüfen und gleichzeitig nach Alternativen zu schauen. Die Empfehlungen würden jedoch erst nach Rücksprache mit dem eigenen Arzt übernommen.

Die vorliegenden empirischen Studien können zwar aufgrund der meist querschnittlichen Untersuchungsanlage keine Langzeitwirkungen attestieren, weisen jedoch insgesamt darauf hin, dass dem Internet aus Usersicht als Informationsquelle zu Gesundheit und Krankheit eine unmittelbare, alltagsrelevante Bedeutung zukommt.

\section{Qualität von Gesundheitsinformationen im Internet}

\subsection{Bestimmung des Qualitätsbegriffs}

Qualität von Medieninhalten wird ebenso vielfältig definiert, wie es Versuche gibt, Aspekte und Ebenen von Qualität zu systematisieren (z. B. Quandt, 2004). Qualität ist keine Eigenschaft eines Produktes oder Medien-Angebotes, sondern sie stellt eine Eigenschaft der Beziehung zwischen Angebot und Beurteilendem dar (vgl. Neuberger, 2004). Dementsprechend wird Qualität von der International Organization for Standardization (1994) definiert als „The totality of characteristics of an entity that bear on its ability to satisfy stated and implied needs“. Qualitätsurteile sind abhängig von dem Wertesystem, aus dem sie abgeleitet sind. Dies können z. B. Werte der Profession oder ihrer unterschiedlichen Schulen, Werte der allgemeinen Ästhetik oder des Publikums sein (Schatz \& Schulz, 1992).

Bei der Qualitätsbeurteilung journalistischer Angebote kann man zwischen zwei Ebenen unterscheiden: Auf der einen Seite steht die Perspektive derjenigen, die z. B. als Publikum, Werbekunden oder Gegenstand der Berichterstattung von den Auswirkungen des Journalismus betroffen sind. Auf der anderen Seite werden die Inhalte und ihre Wirkungen von gesellschaftlichen Repräsentanten mit entsprechender Expertise aus z. B. Wissenschaft, Politik, Journalismus oder Justiz bewertet (Neuberger, 2004). Übertragen auf das vorliegende Forschungsinteresse können entsprechend als zentrale Beurteilungsebenen zum einen Laien, die im Netz Gesundheitsinformationen suchen, und zum anderen Vertreter des Medizinsystems definiert werden, die Informationen unter fachlichen Gesichtspunkten einschätzen und hinsichtlich ihrer Angemessenheit für das Zielpublikum einordnen können. Da es eine eindeutige Bestimmung des Qualitätsbegriffs nicht gibt, wird das Konstrukt in der Regel in seine Bestandteile zerlegt, die jeweils weiter ausdifferenziert werden. Hierdurch wird das Spektrum derjenigen Merkmale definiert, an denen publizistische Qualität gemessen wird. Diese Kriterien werden dann aus der individuellen Perspektive des Betrachters auf ihre Bedeutsamkeit und ihr Vorliegen hin beurteilt. Gemessen wird damit, welchen Qualitätsaspekten welche Relevanz beigemessen wird, d. h., auf welchen Dimensionen das Medienangebot als qualitativ hoch- oder minderwertig eingeschätzt wird. ${ }^{1}$

1 Eine auf die Bewertung der Qualität von Informationsangeboten im Internet zugeschnittene Dimensionierung haben Dahinden, Kaminski und Niederreuther (2004) vorgestellt. Die Auto- 
Qualitätsansprüche haben letztlich immer einen normativen und subjektiven Charakter (vgl. Vlasic, 2004). So finden wir auch bei der Diskussion um die Qualität von Gesundheitsinformationen und die angelegten Kriterien eine Vermischung normativer (im Sinne wünschenswerter und der gesellschaftlichen Verantwortung der Journalisten Rechnung tragender) Ansprüche mit empirisch ermittelten Qualitätskriterien im Sinne einer z. B. durch die Rezipienten zu bestimmenden Größe.

Verglichen mit dem Forschungsstand zur Qualität im Internet im Allgemeinen (vgl. Beck, Schweiger \& Wirth, 2004) ist die spezifischere Debatte zum Thema Qualität von Gesundheitskommunikation im Internet bereits in vollem Gange (Abschnitt 3.2). Zur Messung der Qualität von Gesundheitsinformationen im Internet wurden bereits zahlreiche Kriterienkataloge definiert. Für die vorliegende Studie haben wir in Anlehnung an eine Systematik von Dahinden et al. (2004, vgl. Fußnote 1) eine Synopse dieser Kataloge erstellt (Abschnitt 3.3) und zur Qualitätsmessung herangezogen, indem wir sie Laien und Experten zur Bewertung der drei untersuchten Webseiten (vgl. hierzu Abschnitt 5.1) vorgegeben haben.

\subsection{Tendenzen und Probleme der Qualitätsbeurteilung}

Evaluationsstudien geben zu der Befürchtung Anlass, dass die Qualität von Gesundheitsinformationen im Netz unzureichend ist (Eysenbach et al., 2002). Problematisch sei, dass die Informationsqualität extrem schwanke (Eysenbach \& Diepgen, 1998). Insbesondere bezüglich der Vollständigkeit und Richtigkeit der Informationen wird häufig ein negatives Fazit gezogen (z. B. Griffiths \& Christensen, 2000; Impicciatore et al., 1997). Informationen sind zudem häufig nicht wissenschaftlich abgesichert und irreführend (Fox et al., 2000). Auch die Stiftung Warentest empfiehlt dringend, individuelle medizinische Beratung im Internet mit Vorsicht zu genießen (o. V., 2003). Laut Fox et al. (2000) können Patienten sich selbst nachhaltig schädigen, wenn sie einzelne Aspekte aus dem Kontext isolieren und Empfehlungen annehmen, ohne eine Expertenmeinung hinzuzuziehen und sich über die Güte der Quelle und Informationen rückzuversichern.

Qualitätsdefizite von Gesundheitsinformationen sind jedoch kein internetspezifisches, sondern ein generelles Problem massenmedialer Kommunikation (Hautzinger, 2004). Im Internet wird die Qualitätsfrage jedoch vergleichsweise relevanter. Denn im virtuellen Raum stehen qualitativ hoch- und minderwertige Informationen unmittelbar nebeneinander, Informationen können uneingeschränkt von jedermann online gestellt und damit auch jedem zugänglich gemacht werden. Durch die Niederschwelligkeit des Mediums werden Fehlinformationen zudem leichter verbreitet, und Nutzer, denen die Expertise zur inhaltlichen Bewertung der ggf. speziell an eine andere Zielgruppe gerichteten Informationen meist fehlt, laufen Gefahr, diese falsch einzuordnen (Herbenstreit \& Güntert, 2001).

Neben den überaus kritischen Beurteilungen der Qualität von Gesundheitsinformationen im Internet gibt es jedoch auch Hinweise darauf, dass sich die Informationsqualität im Internet bereits verbessert hat (Pandolfini \& Bonati, 2002). Und inzwischen gibt

ren haben zahlreiche zur Messung klassischer Medieninhalte sowie für das Internet verwendete konkrete Qualitätskriterien gesammelt und zu den vier abstrakteren Dimensionen Inhaltsqualität, technische Qualität, Darstellungsqualität und Interaktionsqualität verdichtet. Die Kriterien wurden so formuliert, dass sie aus unterschiedlichen Perspektiven zu beurteilen sind und damit eine Vergleichbarkeit der Qualitätsbeurteilungen ermöglichen. 
es eine Reihe von Dachverbänden, die Qualitätsstandards, Lizenzierungen und Gütesiegel wie den HON-Code oder das afgis-Logo anbieten, an denen sich die User orientieren können. Die hier angelegten Qualitätsstandards decken sich im Wesentlichen mit den in Abschnitt 3.3 zusammengetragenen Qualitätskriterien. Neben solchen Evaluations- und Lizenzierungsstrategien versucht man, die User durch Empowerment-Strategien, z. B. in Form des DISCERN-Instruments, zu einer eigenständigen Beurteilung von Gesundheitsinformationen zu befähigen (vgl. hierzu ausführlich Hautzinger, 2003, 2004).

In Studien zur Messung der Qualität von Gesundheitsinformationen im Internet werden meistens einzelne Experten herangezogen, deren Urteile nicht ohne weiteres als zuverlässige Indikatoren gelten können. So konnten Craigie et al. (2002) nachweisen, dass die Reliabilität der Expertenurteile überaus gering und damit die Gültigkeit bzw. Verallgemeinerbarkeit dieser Urteile problematisch ist.

Zwar gibt es diverse Versuche, die Qualität gesundheitsbezogener Webseiten zu evaluieren, jedoch haben sich bislang keine einheitlichen und robusten, die Bedürfnisse der Öffentlichkeit bzw. interessierter Laien treffenden Kriterien etabliert. Fast jede Evaluation kommt zu unterschiedlichen Rankings und Testsiegern. Außerdem wurden in den vorliegenden empirischen Studien User- und Expertenurteile nicht verglichen (Ambre et al., 1997; Hägele \& Leopold, 2004).

\subsection{Dimensionen der Qualitätsbeurteilung}

Es gibt bereits einige Versuche, die zahlreichen in der Forschung zur Qualität der Gesundheitskommunikation im Netz verwendeten Indikatoren auf überschaubare Sets an Kriterien zu reduzieren. In einer Metaanalyse haben Kim et al. (1999) aus 29 RatingTools und Publikationen zur Beurteilung von Gesundheitsinformationen im Internet die 165 gemessenen Kriterien zu zwölf spezifischen Kategorien verdichtet. ${ }^{2}$ Ebenfalls eine Metaanalyse von 79 Studien, die sich mit Gesundheitswebseiten befassen und in den Einzelergebnissen 86 Qualitätskriterien eruieren, haben Eysenbach et al. (2002) durchgeführt. ${ }^{3}$ Auch Ambre et al. (1997) haben die aus Expertensicht relevantesten Dimensionen zusammengetragen. ${ }^{4}$

Die eEurope 2002 Initiative der Europäischen Kommission hat 2002 ein Set von Qualitätskriterien definiert, auf das sich private und öffentliche Akteure aller Mitgliedsstaa-

2 Folgende Kriterien werden bei der Qualitätsbeurteilung am häufigsten angewendet: [1] Inhalt der Webseite (Zuverlässigkeit, Genauigkeit, Gültigkeitsbereich und Tiefe der Informationen), [2] Design und Ästhetisches (Layout, Interaktivität, Präsentation, Anmutung, grafische Darstellung, Multimedialität), [3] Offenlegung der Autoren, Sponsoren und Verantwortlichen (Ziel der Webseite, Art der Organisation, Finanzierungsquellen, Herkunft, Autoren/Quellen), [4] Aktualität der Informationen, [5] Seriosität und Glaubwürdigkeit der Quellen, [6] Bequemlichkeit der Nutzung (Usability, Navigation, Funktionalität), [7] Zugang und Verfügbarkeit, [8] interne und externe Verlinkung, [9] Darlegung der Referenzen, Evidenz der Befunde, [10] angestrebte Zielgruppe/Angemessenheit der Informationen für bestimmte Zielgruppen, [11] Kontaktadresse oder Feedback-Optionen, [12] Möglichkeit der Anforderung von Unterstützung durch den User/Dokumentation für den User (Kim et al., 1999).

3 Als wichtigste Qualitätskriterien stellen die Autoren [1] Richtigkeit der Inhalte, [2] Vollständigkeit, [3] Verständlichkeit, [4] Design, [5] Offenlegung der Sponsoren, Eigentümer, Autoren und Referenzen heraus (Eysenbach et al., 2002).

4 Ambre et al. (1997) haben folgende aus Expertensicht besonders wichtige Dimensionen extrahiert: [1] Zuverlässigkeit, [2] inhaltlicher Anspruch, [3] Transparenz, [4] interne und externe Verlinkung, [5] Design, [6] Interaktivität, [7] Warnungen. 
ten verständigen können sollen (Commission of the European Communities, 2002). Die vorgestellten Kriterien sind primär an den Aufgaben der Anbieter als verantwortungsvolle Kommunikatoren orientiert und sollen sowohl für Webangebote gelten, die nur Informationen zur Verfügung stellen, als auch für Interaktionsplattformen. Über allen Kriterien steht die Frage, welche Inhalte für welche Nutzergruppen wie aufbereitet werden sollten, um eine den Bedürfnissen der User angemessene Gesundheitsinformation und bestmögliche Aufklärung betreiben zu können (vgl. hierzu auch Rimal \& Adkins, 2003)..$^{5}$

Wir haben diese Kriterienkataloge zur Beurteilung der Qualität von Gesundheitsinformationen im Internet (vgl. Fußnote 3 bis 6) zunächst gebündelt und auf ein einheitliches Abstraktionsniveau gebracht. Diese Synopse ließ sich in die empirisch bewährte Klassifikation von Dahinden et al. (2004, vgl. auch Fußnote 2) integrieren und zu den vier Dimensionen Inhaltsqualität, technische Qualität, Darstellungsqualität und Interaktionsqualität verdichten. Für uns fungiert diese für Online-Informationsangebote allgemeine Dimensionierung damit als Systematisierungshilfe für die zur Messung der Qualität von Gesundheitsinformationen im Internet herangezogenen konkreten Indikatoren. So ist gewährleistet, dass zum einen konkrete gegenstandsspezifische Kriterien der Qualitätsbeurteilung von Gesundheitsinformationen gemessen werden und dass diese zum anderen an die Forschung zur Qualität im Internet im Allgemeinen anschlussfähig sind. Die folgende Systematik stellt die Ergebnisse der Verdichtungsschritte dar.

1. Inhaltsqualität:

Genauigkeit und Richtigkeit (Evidenz der Befunde);

Vollständigkeit;

Verständlichkeit der Informationen für die Zielgruppe;

Relevanz und Nützlichkeit der Informationen;

regelmäßige und gekennzeichnete Aktualisierung;

Transparenz: Offenlegung der Quellen, Autoren, Referenzen, Sponsoren und Verantwortlichen, Finanzierungsquellen, der Kriterien der Auswahl der Inhalte zur Veröffentlichung, der Ziele der Webseite, der Informationen, die über den User gesammelt werden; Zitation von Originalquellen.

2. Darstellungsqualität:

Design und Ästhetisches: Layout, Anmutungsqualität, grafische Darstellung;

Navigation, Funktionalität (z. B. Übersichtlichkeit, Auffindbarkeit);

Strukturiertheit, logischer Aufbau.

3. Technische Qualität:

Sicherheit;

Ladezeiten;

Suchfunktionen;

Übertragungszeiten.

4. Qualität der Interaktivität:

interne und externe Verlinkung;

Möglichkeit zum Feedback, zur Kontaktaufnahme, der Anforderung von Unterstützung; Zugang zu Foren oder Chat-Rooms;

5 Als zentrale Kriterien wurden definiert: [1] Transparenz: Definition der Zielgruppe, des Kommunikationsziels und Offenlegung der Sponsoren und Eigentümer, [2] Quellennachweise, [3] Sicherheit bei der Übertragung persönlicher Daten, [4] Aktualität, [5] Verantwortlichkeit (für externe Links und Inhalte), [6] Erreichbarkeit (Commission of the European Communities, 2002). 
Transaktionen (Bestellung von Informationsmaterial und Waren). Ausgehend von dem hier entwickelten Kriterienkatalog wird im Folgenden anhand verschiedener Studien analysiert, welche der Qualitätskriterien von Usern wie bewertet und gewichtet werden.

\subsection{Bewertung und Gewichtung von Qualitätskriterien durch die User}

Es stellt sich die Frage, welche der meist von Experten und Vertretern des Gesundheitswesens definierten Qualitätskriterien (vgl. Abschnitte 3.2 und 3.3) für die User in der konkreten Nutzungssituation überhaupt relevant sind und welche Aspekte für die Bewertung einer Gesundheits-Webseite ausschlaggebend sind. Eysenbach und Köhler (2002) konnten im Hinblick auf die Inhaltsqualität zeigen, dass Webseiten dann für vertrauenswürdig gehalten werden, wenn sie von offiziellen Institutionen stammten, verständlich und professionell geschriebene Texte sowie eine Bezugnahme auf wissenschaftliche Quellen aufwiesen. Die Einschätzung der Glaubwürdigkeit als zentralem Aspekt der Inhaltsqualität hängt dabei wesentlich von den Gesundheitsvorstellungen und dem Gesundheitsbewusstsein sowie von der Soziodemografie der User ab (DuttaBergman, 2003). Und die Beurteilung der Inhaltsqualität ist schon allein deshalb höchst subjektiv, da die Einstellung zu medizinischen Informationen (z. B. zu Therapiemethoden wie Akupunktur) selbst in Fachkreisen erheblich divergiert. Im Hinblick auf die Darstellungsqualität wurden der leichte Zugang zu den Informationen und die Übersichtlichkeit der Seiten, ein professionelles Layout, angebotene Suchmöglichkeiten auf der Seite, eine benutzerfreundliche und klar strukturierte Benutzeroberfläche als wichtig beurteilt (ebd.). Auch die Ladezeiten (also die technische Qualität) wurden als Kriterium für die Beurteilung einer Gesundheitswebseite genannt.

Das Kriterium der Interaktionsqualität hat sich in den bisherigen empirischen Studien als weniger beurteilungsrelevant erwiesen (Dahinden et al., 2004). Das mag bezogen auf den konkreten Forschungsgegenstand u. a. daran liegen, dass die derzeit angebotenen inhaltlich breit angelegten und an Laien gerichteten Webseiten mit Gesundheitsinformationen nur vergleichsweise wenige Möglichkeiten der unmittelbaren Interaktion bieten. Kontakt zu Experten und Foren haben auf den Portalen nachrangigen Stellenwert, und die User suchen eher nach Informationen in Form von Fachtexten als nach interaktiven Angeboten (Fox et al., 2000). Deshalb wird das Kriterium der Interaktionsqualität im Folgenden vernachlässigt.

Entscheidend dafür, welche Kriterien für die Qualitätsbeurteilung ausschlaggebend sind, ist neben den erwähnten Faktoren das Ausmaß der individuellen Betroffenheit. Wer weniger in ein Gesundheitsthema involviert ist, der lässt sich eher von Design und Ästhetik einer Webseite beeinflussen. Thematisch Interessierte und Betroffene achten stärker auf den Inhalt und die Aktualität der Informationen (Barnes et al., 2003). Eine Studie von Dutta-Bergman (2004) zeigt, dass die Kriterien der Qualitätsbeurteilung innerhalb einer Dimension einander beeinflussen. So hat die Vollständigkeit der dargebotenen Informationen einen positiven Einfluss auf die wahrgenommene Glaubwürdigkeit der Webseite. Dieser Effekt ist bei denjenigen stärker ausgeprägt, die „nur“ surfen, als bei denjenigen, die gründlich nach bestimmten Informationen suchen.

Qualitätsanspruch und tatsächliche Beurteilungsstrategien scheinen jedoch auseinander zu fallen. In verschiedenen Studien wurde gezeigt, dass Urheber und Herkunft der Informationen für die Probanden entgegen ihren eigenen Angaben letztlich doch eher unerheblich waren (Barnes et al., 2003; Eysenbach \& Köhler, 2002). Bei der allgemeinen Einschätzung wurden inhaltliche Aspekte für besonders wichtig gehalten. Von geringe- 
rer Bedeutung waren Bequemlichkeit der Nutzung, Design und Anmutungsqualitäten. Bei der konkreten Beurteilung einzelner Seiten erwiesen sich hingegen gerade die letztgenannten Kriterien als relevant.

Zusammenfassend können wir festhalten, dass für die Onlinekommunikation gesundheitsbezogener Inhalte die inhaltlich richtige (auf Evidenzen beruhende), vollständige und verständliche Vermittlung der Inhalte, also die Inhaltsqualität von besonderer Bedeutung ist (Commission of the European Communities, 2002; Eysenbach \& Köhler, 2002; Eysenbach et al., 2002; Kim et al., 1999). Das Gleiche gilt für die Aufbereitung der Inhalte bzw. Darstellungsqualität. Denn auch bei korrekter inhaltlicher Darstellung können User die Inhalte nur bei einer für medizinische Laien nachvollziehbaren Struktur und Aufbereitung der Webseite effektiv nutzen. Die technische Qualität ist insofern von erheblicher Wichtigkeit, als die Webseiten bei verschiedenen PC-Ausstattungen schnell und zuverlässig laufen müssen. Außerdem sind gerade im Rahmen medizinischer und gesundheitsbezogener Onlinekommunikation die Privatsphäre und Sicherheit der User von großer Bedeutung. Die drei genannten Qualitätskriterien und ihre wichtigsten Indikatoren werden in der folgenden empirischen Studie (vgl. Abschnitte 4 bis 6) untersucht.

\section{Ableitung der Forschungsfragen}

Die zentrale Fragestellung der Studie bezieht sich auf die Qualitätsbewertung ausgewählter deutschsprachiger Webseiten durch ihre User. In diesem Zusammenhang sollen die Gewichtung verschiedener Qualitätskriterien, ihre Determinanten und die Bedeutung der Bewertung für die Weiternutzung der Webseite untersucht werden. Außerdem soll verglichen werden, in welcher Beziehung die Urteile von Usern und Experten stehen.

Die zu untersuchenden Qualitätsdimensionen Inhaltsqualität, Darstellungsqualität und technische Qualität wurden aus den bisherigen Studien zur Qualität im Internet und zur Qualität von Gesundheitsinformationen im Internet abgeleitet. Bei der Bewertung gehen wir von zwei unterschiedlichen Nutzungssituationen und -motiven aus, in denen sich die User befinden können: Sie verschaffen sich entweder einen allgemeinen Eindruck von einer Webseite und surfen ungerichtet, oder sie haben ein bestimmtes Problem bzw. einen konkreten Suchauftrag, den sie verfolgen (vgl. Dutta-Bergman, 2004). Diese beiden Nutzungssituationen können unabhängig voneinander auftreten, aber auch zeitlich nah aufeinander folgen, z. B. wenn die User vor der Beantwortung einer spezifischen Frage zunächst versuchen, sich ein allgemeines Urteil über die Webseite zu bilden und davon die Weiternutzung abhängig machen.

FF 1: Gibt es signifikante Unterschiede zwischen der Bewertung der Qualität einer Webseite nach einer ersten unspezifischen Sichtung der Seite und nach einer spezifischen (krankheitsbezogenen) Recherche?

In den theoretischen Vorarbeiten der obigen Abschnitte wurde herausgestellt, dass bestimmte personenbezogene Variablen bei der Erfahrung im Umgang mit Gesundheitswebseiten und auch bei ihrer Bewertung eine Rolle spielen können (vgl. Abschnitte 2 und 3.4). Insbesondere Frauen sind an Gesundheitsthemen interessiert und können als "Gesundheitsmanagerinnen" der Familie angesehen werden (Dutta-Bergman, 2003).

FF 2: Inwiefern determinieren Eigenschaften der User wie das Geschlecht, das Interesse an Gesundheitsthemen oder die Internetaffinität die Qualitätsbewertung?

Für die wissenschaftliche und praktische Arbeit mit Gesundheitswebseiten ist es von Bedeutung, welche Qualitätskriterien letztendlich ausschlaggebend für die Gesamtbe- 
wertung und Weiternutzung der Seiten sind (vgl. Abschnitte 2 und 3.4). In verschiedenen Studien wurde herausgestellt, dass User ihr Gesundheitsverhalten häufig nach den im Internet gefundenen Informationen ausrichten (Emnid, 2003; Fox et al., 2000). Dementsprechend soll überprüft werden, welches Gewicht die einzelnen Qualitätsaspekte im Gesamturteil der User erhalten und welchen Einfluss sie auf das künftige Nutzungsverhalten der Gesundheitswebseiten haben.

FF 3: Welche Bedeutung kommt einzelnen Qualitätsdimensionen für das Gesamturteil zu?

FF 4: Inwiefern sind die Qualitätsurteile relevant für die zukünftige Nutzung der Webseite?

Schließlich soll untersucht werden, inwieweit die Urteile von Usern mit denen ausgewiesener Experten korrespondieren.

FF 5: In welchem Verhältnis stehen die Urteile der User zu Experteneinschätzungen der Qualität der Webseiten?

Die fünf Forschungsfragen wurden im Rahmen einer User-Befragung im Online-Labor und einer Expertenbefragung überprüft. Die empirische Umsetzung der Studien, die untersuchten Webseiten und Inhalte sowie Merkmale der Stichprobe werden im Folgenden dargestellt.

\section{Empirische Studie}

\subsection{Die im Rabmen der Studie untersuchten Webseiten: Lifeline.de, Netdoktor.de und Gesmed.ch}

Kriterien für die Auswahl der Webseiten waren, dass sie eine hinlängliche Reichweite haben, im Bereich Health Content angesiedelt sind und sich vornehmlich an nicht-wissenschaftlich oder -klinisch geschulte User richten. Vor diesem Hintergrund wurden Lifeline.de, Netdoktor.de und Gesmed.ch ausgewählt. Laut Cramer und Stiehler (2000) gehören die drei untersuchten Webseiten zu den bekanntesten im deutschsprachigen Raum. Inhaltlich decken sie ein sehr ähnliches, an allgemeinen Gesundheitsthemen und spezifischen Hintergrundinformationen ausgerichtetes Spektrum ab. Bezüglich der technischen Ausstattung gibt es keine relevanten Unterschiede zwischen den Webseiten. Die HTML-Seiten sind ähnlich groß (von 20 bis $50 \mathrm{~KB}$ ), und die Webseiten haben dementsprechend vergleichbare Ladezeiten. Alle befragten Anbieter gaben an, dass sie eigene Server hätten, die direkt an den Provider angebunden seien.

Alle Webseiten arbeiten kommerziell. Wesentliche Unterschiede lassen sich in der Art der Aufmachung und der Tiefe der Informationen feststellen. ${ }^{6}$ Lifeline.de ist werbefinanziert und bei der IVW gelistet. Hiernach verzeichnet die Webseite 500000 Visits und 2,6 Mio. Page Impressions (PIs). ${ }^{7}$ Lifeline.de ist dominiert von vornehmlich aktuellen Themen, wohingegen die Hintergrundinformationen zu Krankheiten und die Inhalte des Lexikons eher im Hintergrund stehen. Angeboten werden Gewinnspiele, eine lange Liste von Selbsttests und ein Forum zu verschiedenen Rubriken. Netdoktor.de ist ebenfalls durch Werbung finanziert und bei Gallup gelistet. Die Webseite kommt auf circa 400000 Visits und 4,1 Mio. Page Impressions pro Monat (persönliche Mitteilung

6 Auf umfassende Informationen und Screenshots zu den Webseiten muss hier aus Platzgründen verzichtet werden, diese können jedoch bei den Autoren angefordert werden.

7 Die Nutzerdaten zu allen drei Webseiten wurden im Vorfeld der beiden Studien im April bis Juni 2003 recherchiert. Alle Angaben beziehen sich auf diesen Untersuchungszeitraum. 
Ute Vits, netdoktor.de, 30. Mai 2003). Netdoktor.de bietet die Rubriken Aktuelles (z. B. zu SARS), Communities, ein Lexikon, Interaktion (Expertenrat), Ratgeber und Service (z. B. Arzt finden) an. Auffallend ist, dass Fachärzte als Berater tätig sind, eine eigene Redaktion beschäftigt wird und Netdoktor sich dem „Health On Net Code“ verpflichtet. Gesmed.ch ${ }^{8}$ wird im Rahmen des Scout24-Portfolios angeboten und ist ebenfalls werbefinanziert. Die Seite verzeichnet circa 500000 Visits und 2,6 Mio. Page Impressions monatlich (persönliche Mitteilung gesmed.ch, 30. Mai 2003). Zentrale Rubriken sind Reise, ein Lexikon, Ratgeber, Informationen zu einzelnen Krankheiten.

\subsection{Die Nutzerbefragung im Online-Labor: Durchfübrung, Fragebogen und Stichprobe}

Im Rahmen der Laborstudie wurden Internetnutzer zu ihrer Bewertung inhaltlicher und formaler Aspekte befragt. Sie wurden über Aushänge an der Universität Hamburg und in umliegenden Supermärkten rekrutiert und erhielten ein Honorar in Höhe von $15 €$. Die Befragung ist also nicht repräsentativ. Voraussetzung für die Teilnahme war, dass die Befragten „regelmäßig das Internet nutzen“. Die Befragung fand im September 2003 an der Universität Hamburg in einem Online-Labor mit 13 Plätzen statt. Die Befragten wurden gebeten, einen aus vier Teilen bestehenden Fragebogen auszufüllen.

Der Fragebogen enthielt im ersten Teil allgemeine Fragen zur bisherigen Nutzungserfahrung mit Gesundheitswebseiten. Zu Beginn des zweiten Teils erhielten die Probanden die Instruktion, den Bildschirm anzuschalten, auf den bereits aufgerufenen drei Webseiten zu surfen und sie anhand von elf allgemeinen Fragen zu bewerten (z. B. Fragen danach, ob man die Webseiten an Bekannte weiterempfehlen würde und ob man sich auf dieser Webseite Rat suchen würde). An dieser Stelle wurden auch Fragen zur technischen Qualität gestellt (z. B. zu Ladezeiten und der Sicherheit der Webseite). Dabei stand der intuitive Eindruck der User im Vordergrund. Das Tempo konnten die Befragten selbst bestimmen.

Der dritte Teil des Fragebogens wurde mit der Aufforderung eingeleitet, alle drei Webseiten bezüglich der Inhalte zu einer der drei Krankheiten Bandscheibenvorfall, Depression oder Diabetes zu bewerten. ${ }^{9}$ Die Bewertung fand anhand vorgegebener Fragen statt. Den Befragten stand es frei, vor oder während der Beantwortung die notwendigen Informationen auf der Webseite zu recherchieren. Zum einen wurden die Probanden befragt, ob die Entstehung, Erkennung, die Symptome und Therapie der Krankheiten ihrer Ansicht nach gut und korrekt dargestellt waren (Inbaltsqualität der klinischen Kriterien) und zum anderen, wie verständlich, vollständig und einfach sie dargestellt waren (Inhaltsqualität der formalen Kriterien). Außerdem bewerteten die User die Darstellungsqualität, operationalisiert über die Bewertung der Navigation und des Designs. Die Zeit für die Beantwortung konnte wiederum von den Befragten selbst bestimmt werden. Abschließend, im vierten Teil des Fragebogens, wurden die User gebeten, ein Gesamturteil zu den Webseiten abzugeben, und es wurden die Nutzung von Fachzeitschriften, Fernsehsendungen und des Internets für Themen im Bereich Krankheit und Gesundheit

8 Gesmed.ch wurde nach der Untersuchung zunächst zu Gesundheitsscout24.ch und ist jetzt unter Sprechzimmer.ch erreichbar.

9 Jeweils ein Drittel der Stichprobe wurde zu einer der drei Krankheiten befragt. Diese Krankheiten wurden ausgewählt, weil sie sehr verbreitet sind (American Psychiatric Association, 2003: 402/403; Anderson, 1991; Herold, 2004). In den späteren Berechnungen werden die Krankheiten nur noch bei einer deskriptiven Auswertung der Ergebnisse berücksichtigt. 
sowie die Mediennutzung und soziodemografische Daten abgefragt. Die Befragung dauerte durchschnittlich circa 50 Minuten.

Die Stichprobe umfasst 101 Personen. Insgesamt 61 männliche und 40 weibliche Personen nahmen an den Untersuchungen teil. Das Durchschnittsalter betrug 28 Jahre (Min $=18, \operatorname{Max}=73)$. Die Mehrzahl der Befragten verfügte über einen formal hohen Bildungsabschluss (28 Befragte mit Hochschul- oder Fachhochschulabschluss, 53 Befragte mit Abitur bzw. Vordiplom, acht Befragte mit Real- bzw. Handelsschulabschluss oder Fachabitur, zwei Personen mit Hauptschulabschluss und zwei mit Lehre als höchstem Abschluss). 74 Personen befanden sich noch in der Ausbildung (Studium, Schule oder Lehre), 22 arbeiteten als Angestellte oder Freiberufler, fünf waren ohne Beschäftigung oder Rentner.

Insgesamt 96 Personen verfügten bei sich zu Hause oder an ihrer Arbeitsstelle über einen Internetanschluss, den sie regelmäßig nutzen konnten. Im Durchschnitt verbrachten die Befragten 1,4 Stunden pro Tag im Internet. Das ist verglichen mit der durchschnittlichen täglichen Internetnutzungsdauer der deutschen Onlinenutzer (2,3 Stunden) eher wenig (vgl. Media Perspektiven, 2003). 45 Befragte nutzten mindestens einmal monatlich Fernsehsendungen, um sich über Themen im Bereich Gesundheit und Krankheit zu informieren, 26 Personen nutzten hierzu das Internet und 17 Fachzeitschriften. Diese Ergebnisse sind mit den Ergebnissen anderer Studien vergleichbar: In einem Panel, das repräsentativ für die Käufer rezeptfreier Medikamente ist, zeigt sich, dass 35 Prozent der Befragten TV-Sendungen, 41 Prozent Fachzeitschriften und 35 Prozent das Internet nutzen (BSMO, 2003).

\subsection{Die Expertenbefragung: Durchführung, Kriterienkataloge und Stichprobe}

Sieben Experten beurteilten die Inhaltsqualität der Webseiten hinsichtlich der Krankheiten Diabetes, Bandscheibenvorfall oder Depression. Damit wurde hier im Gegensatz zu den meisten vorherigen Studien mehr als ein Experte pro Krankheit befragt, und es ist möglich, die Übereinstimmung in den Qualitätsurteilen zu berechnen (vgl. Abschnitt 6). Dieses Vorgehen entspricht den Forderungen in der Literatur zur Evaluation von Gesundheitsinformationen im Netz (Eysenbach \& Diepgen, 1998). Dennoch handelt es sich bei der vorliegenden Studie nicht um eine repräsentative Befragung, die Experten wurden willkürlich nach ihrer Verfügbarkeit ausgewählt.

Die Experten nahmen zu einem Kriterienkatalog Stellung und beurteilten (dichotom) für jede der drei Webseiten, ob die wesentlichen Inhalte vollständig und richtig abgebildet wurden. In der Metaanalyse von Eysenbach et al. (2002) zeigt sich, dass das Ausmaß der eingeschätzten Genauigkeit (accuracy) der Gesundheitsinformationen vom Beurteilungskriterium abhängig ist. Drei verschiedene Strategien dominieren: Wenn kein standardisiertes Kriterium benannt war oder die persönliche Meinung des (wie auch immer qualifizierten) Beurteilenden ausschlaggebend war, wurden nur gut 15 Prozent der Webseiten in ihrer Information als ungenau eingeschätzt, unter Rückgriff auf klinische Leitlinien sogar 38 Prozent. Die Qualitätsbeurteilung variiert also stark mit dem Kriterium, an dem die Einschätzung festgemacht wird.

Zur Beurteilung der inhaltlichen Qualität der drei Webseiten im Themengebiet Diabetes wurden ein praktizierender Endokrinologe und Diabetologe des Universitätsspitals Zürich und ein niedergelassener Internist und Diabetologe aus Herne befragt. Sie bewerteten die drei Angebote unabhängig voneinander nach einem systematischen Beurteilungsraster, zunächst allgemein zur Darstellung der Klassifikation, Epidemiologie und Komplikationen. Daraufhin wurden die Experten gebeten, für Typ-1-Diabetes und Typ- 
2-Diabetes die korrekte und vollständige Darstellung der Häufigkeit der Krankheit, ihrer Ursachen, Vererbung, Prävention, der Symptome, Diagnose, Therapie, ratsamer Kontrollen, der Prognose bei Krankheitsausbruch und die Zukunftsperspektiven zu bewerten.

Die drei Webseiten wurden in Bezug auf das Themengebiet Bandscheibenvorfall nach einem Kriterienkatalog beurteilt, der von zwei Physiotherapeutinnen aus dem Raum Zürich erarbeitet wurde. Bewertet wurden wiederum die Ursachen (Pathologie), die Symptome (Schmerz, Ausfälle in den Bereichen Motorik und Sensorik, Inkontinenz), der typische Verlauf, die Therapiemöglichkeiten (Medikamente, Physiotherapie, Chiropraktik, Training, Operation, Ergotherapie) und die Prognose der Krankheit. Drei weitere praktizierende Physiotherapeutinnen beurteilten, ob die Webseiten im Hinblick auf die Kriterien vollständig und richtig waren. Alle Physiotherapeutinnen praktizieren seit mindestens fünf Jahren und behandeln täglich Patienten mit Rückenproblemen.

Zwei Wissenschaftler des Fachbereiches Psychologie der Universität Hamburg mit dem Schwerpunkt klinische Psychologie haben die Webseiten hinsichtlich des Störungsbildes Depression bewertet. Der Kriterienkatalog entsprach den Kriterien zur Kategorisierung klinisch-psychiatrischer Störungen in gängigen klinischen Publikationen zum Thema. Bewertet wurden die Vollständigkeit und Richtigkeit der Darstellung, die Klassifikation, Epidemiologie, Komplikationen, Prävalenz, Ursachen und Risiken, Vererbung, Prävention, Symptome, Kontrollen und Erkennung der Krankheit, ihre Diagnose, Therapie und Prognose.

Im Anschluss an die kriterienorientierte Befragung wurden die Experten um einen allgemeinen Eindruck zu den drei Webseiten gebeten. Die Experten erhielten den Kriterienkatalog per E-Mail oder Post, das Durcharbeiten der Kriterienkataloge dauerte nach Auskunft der Experten circa 50 Minuten.

\section{Ergebnisse}

\subsection{Die Qualitätsurteile der User}

Die Unterschiede zwischen der ersten, intuitiven Bewertung einer Seite und dem zweiten, auf einer krankheitsbezogenen Recherche beruhenden Urteil (FF 1) wurden mit T-Tests für abhängige Stichproben berechnet. Verglichen wurden jeweils die Gesamtbewertung der Webseite und die Bereitschaft zur Weiternutzung vor (Bewertung zu Beginn der Befragung) und nach der inhaltlichen Recherche (Bewertung am Ende der Befragung). Die Ergebnisse sind in Tabelle 1 zusammengefasst.

Der Vergleich der Bewertungen zeigt kein grundsätzliches Beurteilungsmuster in dem Sinne, dass der erste Eindruck grundsätzlich besser oder schlechter ist als der auf einer gründlichen Recherche beruhende. Vielmehr ist die Bewertung abhängig von den einzelnen Webseiten. Die eher populär aufgemachten Webseiten Lifeline.de und Gesmed.ch werden nach dem ersten Eindruck besser bewertet als nach einer konkreten Recherche, im Gegensatz dazu bewerten die Befragten die eher informativ aufgemachte Webseite Netdoktor.de besser, wenn sie sich intensiver mit der Webseite und konkreten Inhalten beschäftigt haben. Offensichtlich revidieren also die User im Verlauf der Nutzung ihr Urteil. Das wiederum führt dazu, dass die Bewertungen der Webseiten nach einer spezifischen Recherche stärker polarisieren. Während nach einem ersten Eindruck ähnlichere Urteile abgegeben werden (vgl. zweite Spalte in Tabelle 1), zeigen sich nach einer spezifischen Recherche eindeutiger die Gewinner (Netdoktor.de), das Mittelfeld (Lifeline.de) und die Verlierer (Gesmed.ch) (vgl. dritte Spalte in Tabelle 1). 
Tabelle 1: Mittelwertsunterschiede zwischen der Gesamtbewertung der Webseiten vor und nach einer krankheitsspezifischen Recherche*

\begin{tabular}{lcccc}
\hline & $\begin{array}{c}\text { Bewertung vor } \\
\text { spezifischer } \\
\text { Recherche } \\
\text { (arithmet. } \\
\text { Mittel) }\end{array}$ & $\begin{array}{c}\text { Bewertung nach } \\
\text { spezifischer } \\
\text { Recherche } \\
\text { (arithmet. } \\
\text { Mittel) }\end{array}$ & $\begin{array}{c}\text { T-Wert } \\
(\text { df })\end{array}$ & Signifikanz \\
\hline Lifeline.de & 2,74 & 2,91 & $-1,182(94)$ & n. $s$. \\
Gesmed.ch & 2,80 & 3,24 & $-3,701(94)$ & $\mathrm{p}<.001$ \\
Netdoktor.de & 2,30 & 2,00 & $2,608(96)$ & $\mathrm{p}<.010$ \\
\hline
\end{tabular}

* range: $1=$ sehr gut $/$ ja bis $5=$ sehr schlecht/nein

Im Rahmen der zweiten Forschungsfrage (FF 2) soll untersucht werden, ob bestimmte personenbezogene Variablen einen Einfluss auf das Qualitätsurteil haben. Für die Berechnungen des Einflusses der Variablen Geschlecht, Interesse an Gesundheitsthemen und Internetaffinität wurden T-Tests für unabhängige Stichproben gerechnet. Das Interesse an Gesundheitsthemen wurde über die Nutzungsfrequenz von gesundheitsbezogenen Fachzeitschriften, Fernsehsendungen und Internetangeboten operationalisiert. Für die Gruppenbildung wurden die Gesundheitsinteressierten (mindestens monatliche Nutzung der genannten Medien) und die nicht Interessierten (Nutzung seltener als einmal monatlich) unterschieden. Die Internetaffinität wurde über die tägliche Internetnutzungsdauer gemessen. Die Einteilung in zwei Gruppen erfolgte anhand des Medians von $\mathrm{Md}=1$ Stunde. Für die so gebildeten Gruppen sollten die Bewertungen der Inhalts-, Darstellungs- und technischen Qualität gemessen werden. Gruppenbezogene Unterschiede konnten jedoch nicht gefunden werden. ${ }^{10}$

\subsection{Die Bedeutung einzelner Qualitätskriterien für die Gesamtbewertung und Weiter- nutzung einer Seite}

Ausgehend von den oben dargestellten Ergebnissen zur Bewertung der Webseiten ist nun von Interesse herauszufinden, wie die unterschiedlichen Bewertungskriterien gewichtet sind bzw. welche Bedeutung sie für das Gesamturteil der User haben (FF 3). Um diese Frage zu beantworten, wurden alle Analysen getrennt für die drei Webseiten (Lifeline.de, Gesmed.ch, Netdoktor.de) und über die drei untersuchten Krankheiten (Bandscheibenvorfall, Diabetes, Depression) durchgeführt. Die Frage nach der Gesamtbewertung der Webseite macht vor allem vor dem Hintergrund einer gewissen Nutzungserfahrung Sinn, so dass nur die krankheitsbezogenen Bewertungen der Inhaltsund Darstellungsqualität einbezogen wurden.

Vor allem die Darstellung der möglichen therapeutischen Maßnahmen (Lifeline.de und Gesmed.de), die Vollständigkeit der Inhalte (für alle Webseiten) sowie die Navigation (Lifeline.de und Gesmed.ch) spielen eine Rolle für die Gesamturteile der User. Eine Regressionsanalyse, in der die Bewertungen aller Webseiten nach den oben angegebe-

10 Insgesamt wurden über 100 T-Tests und (für die Variable Alter) sechs Korrelationen berechnet, da alle Berechnungen sowohl über alle Webseiten und für die Qualitätsindizes als auch getrennt für die einzelnen Webseiten und Qualitätskriterien durchgeführt wurden. Die einzelnen Ergebnisse werden hier aus Platzgründen nicht dargestellt. 
nen Kriterien untersucht wurde, stellte die Darstellung der Krankheitsentstehung, die Vollständigkeit der Inhalte und die Navigation als signifikante Prädiktoren heraus (vgl. Tabelle 2, vorletzte Spalte).

Im zweiten Schritt (FF 4) wurde berechnet, welchen Einfluss die Qualitätsbewertung der User auf die Weiternutzung der Webseiten hat. Alle Ergebnisse der Regressions-

\section{Tabelle 2: Regressionsanalysen zur Vorbersage der Gesamtbewertung und Weiternut- zung gesundheitsbezogener Webseiten anhand ibrer Inhalts- und Darstel- lungsqualität ${ }^{+}$}

\begin{tabular}{|c|c|c|c|c|c|c|c|c|}
\hline & \multicolumn{2}{|c|}{ Lifeline.de } & \multicolumn{2}{|c|}{ Gesmed.ch } & \multicolumn{2}{|c|}{ Netdoktor.de } & \multicolumn{2}{|c|}{ Alle Webseiten } \\
\hline & 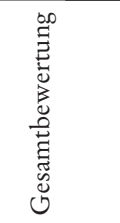 & 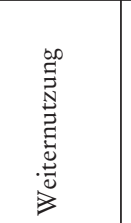 & 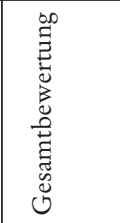 & 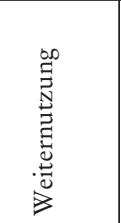 & 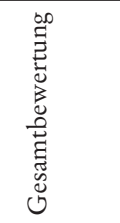 & 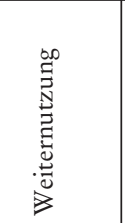 & $\begin{array}{l}\infty \\
\sum_{0}^{\infty} \\
0 \\
0 \\
0 \\
0 \\
0 \\
0 \\
0 \\
0 \\
0 \\
0\end{array}$ & 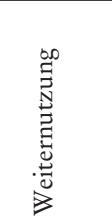 \\
\hline \multicolumn{9}{|c|}{ Inhaltsqualität (klinische Kriterien) } \\
\hline Entstehung & .10 & .22 & $.34 * \%$ & $.34 *$ & .05 & .13 & $.19 \% *$ & .08 \\
\hline Erkennung & .07 & -.26 & -.13 & -.17 & .10 & .17 & .10 & .09 \\
\hline Symptome & .18 & $.36 *$ & .19 & .12 & .10 & -.03 & .14 & .13 \\
\hline Therapie & $.19 *$ & .03 & $.20 *$ & .01 & .15 & .11 & .06 & -.08 \\
\hline \multicolumn{9}{|c|}{ Inhaltsqualität (formale Kriterien) } \\
\hline Verständlichkeit & .06 & -.01 & .01 & -.03 & .08 & $.28 *$ & .04 & -.13 \\
\hline Vollständigkeit & $.38 \% *$ & $.34 *$ & $.33 * *$ & .18 & $.44 * * *$ & -.04 & $.34 *$ & -.06 \\
\hline $\begin{array}{l}\text { Informationen } \\
\text { gut zu finden }\end{array}$ & -.01 & -.07 & .06 & .06 & $.15 *$ & -.14 & .12 & -.01 \\
\hline \multicolumn{9}{|c|}{ Darstellungsqualität } \\
\hline Design & -.07 & $.22 *$ & .00 & .16 & -.02 & .18 & -.10 & -.01 \\
\hline Navigation & $.20 \%$ & $.28 *$ & $.14 *$ & .11 & .12 & $.22 *$ & $.24 \% *$ & $.39 \div * \%$ \\
\hline$R^{2}$ korr & $.823 \% *$ & $.495 \% * \%$ & $.772 \% * *$ & $.295 \% * \%$ & $.692 * * *$ & $.408 * * \%$ & $.655 \%$ & $.146 * \cdots$ \\
\hline
\end{tabular}

analysen befinden sich in Tabelle 2. Ob die Webseite Lifeline.de weitergenutzt wird, hängt demnach vor allem von der Darstellung der Symptome, der Vollständigkeit der Darstellung und den Kriterien Design und Navigation ab. Die Weiternutzung der Webseite Gesmed.ch ist allein abhängig von der Darstellung der Krankheitsentstehung. Die Weiternutzung der Webseite Netdoktor.de wird determiniert durch die verständliche Darstellung und die Navigation auf der Seite. Hier zeigt sich also kein von den unterschiedlichen Webseiten unabhängiges Ergebnis. Es finden sich außer der Navigation (Lifeline.de und Netdoktor.de) keine Qualitätskriterien, die als „General-Prädiktoren“ bei der Vorhersage der Weiternutzung gelten können. Vielmehr ist es von Webseite zu Webseite unterschiedlich, welche Qualitätskriterien eine Vorhersage der Weiternutzung erlauben. Eine Regressionsanalyse, in die Bewertungen aller Webseiten nach den oben angegebenen Kriterien eingingen, bestätigt diesen Eindruck und stellt die Navigation als einzigen relevanten Prädiktor heraus (vgl. Tabelle 2, letzte Spalte). Hier zeigt sich, dass neben der wahrgenommenen Qualität einer Webseite vermutlich auch Routinen, ein in- 
dividueller Bedarf, die persönliche Betroffenheit und der aktuelle Nutzwert eine Rolle spielen. Diese Aspekte wurden als Prädiktoren nicht berücksichtigt und würden vermutlich zur Varianzaufklärung beitragen.

\subsection{User- und Expertenurteile im Vergleich}

In einer zweiten Studie wurden $(\mathrm{N}=7)$ Experten klinische Kataloge zur Bestimmung und Beschreibung der drei Krankheiten Diabetes, Bandscheibenvorfall und Depression vorgelegt, und sie wurden gebeten, ihr Urteil dazu abzugeben, ob diese korrekt und vollständig auf den drei untersuchten Webseiten dargestellt werden (vgl. Abschnitt 5.3). Mit den Ergebnissen der Expertenbefragung soll analysiert werden, ob User und Experten die Inhaltsqualität (klinische Kriterien) unterschiedlich bewerten (FF 5). Auf diese Weise kann bis zu einem gewissen Grad ermessen werden, ob die User fachlich kompetente Urteile fällen. Denn natürlich ist nicht nur von Interesse, ob Laien die Inhalte einer Webseite für gut befinden, sondern auch, ob ihr Qualitätsurteil mit einem fachlich-professionellen Urteil übereinstimmt. Solch ein Vergleich wurde bisher nicht durchgeführt und birgt ungeachtet aller Vorteile eine Reihe von Problemen. Die Experten beurteilen die Inhalte auf den Webseiten zwar nach professionell abgesicherten und in ihrem Fach etablierten Kriterien. Als einen im Sinne garantierter sachlicher Richtigkeit objektiven Indikator können die Expertenurteile jedoch nicht angesehen werden. Es handelt sich lediglich um eine auf der Basis formal abgesicherter, fachlicher Kriterien formulierte Perspektive auf den Gegenstand, die dennoch in sich heterogene Urteile hervorbringen kann. Auch sind Qualitätsansprüche innerhalb eines Faches durchaus sehr unterschiedlich (z. B. Meinungen der Vertreter alternativer Medizin und Schulmedizin zu verschiedenen Therapiemethoden). Unterschiede zwischen Laien- und Expertenurteil müssen deshalb mit großer Vorsicht und immer vor dem Hintergrund der Zusammensetzung beider Stichproben und der Kriterienkataloge interpretiert werden.

Für die Auswertung der fünften Forschungsfrage wurden die Expertenratings ebenso wie die Ratings der User über alle Krankheiten in einem Summenindex zusammengefasst. ${ }^{11}$ Die Urteile der User und Experten sind in Tabelle 3 für die drei Webseiten zusammengefasst. Berechnet ist jeweils das arithmetische Mittel. Die Mittelwerte weisen auf „Gewinner“ und „Verlierer“ hin: Netdoktor.de schneidet besser ab als die anderen beiden Webseiten, und die Inhaltsqualität von Gesmed.ch bewerten sowohl User als auch Experten unterdurchschnittlich.

Die (Pearson-Produkt-Moment) Korrelation der Urteile von Usern und Experten beträgt $r=.77$, ein positives Userurteil korrespondiert also in sehr hohem Maße mit einem positiven Expertenurteil im Hinblick auf die drei untersuchten Qualitätskriterien. Die Experten weisen Übereinstimmungen von $\mathrm{CR}=.80(\mathrm{n}=3)$ in ihrer Beurteilung des Themas Bandscheibenvorfall auf den drei Webseiten auf, CR $=.88(\mathrm{n}=2)$ für Diabetes und CR = .77 ( $\mathrm{n}=2)$ für Depression (CR berechnet nach Früh, 2001: 179). Im Gegensatz zu anderen Studien (vgl. Craigie et al., 2002), zeigt sich hier eine vergleichsweise hohe Übereinstimmung.

11 Da die Experten nur dichotome Beurteilungen abgegeben haben, wurden ihre Urteile zusammengezählt $(0=$ pos., $1=$ neg.) und durch die Anzahl der Urteile dividiert. Als Expertenurteil resultiert also immer ein Wert zwischen 0 und 1. Die Urteile der User haben den Range von 1 (positive Bewertung) bis 5 (negative Bewertung). 
Tabelle 3: Beurteilung der Webseiten Lifeline.de, Gesmed.ch und Netdoktor.de aus Sicht von Usern und Experten im Hinblick auf die Darstellung der Entstebung der untersuchten Krankheiten, ibrer Symptome und Therapie

\begin{tabular}{llll}
\hline Lifeline.de & Entstehung & Symptome & Therapie \\
\hline User-Urteil & $\mathrm{M}=2,49$ & $\mathrm{M}=2,74$ & $\mathrm{M}=2,89$ \\
Experten-Urteil & $\mathrm{M}=0,22$ & $\mathrm{M}=0,22$ & $\mathrm{M}=0,14$ \\
\hline & & & Therapie \\
\hline Gesmed.ch & Entstehung & Symptome & $\mathrm{M}=3,58$ \\
\hline User-Urteil & $\mathrm{M}=2,89$ & $\mathrm{M}=2,74$ & $\mathrm{M}=0,84$ \\
Experten-Urteil & $\mathrm{M}=0,45$ & $\mathrm{M}=0,66$ & \\
\hline & & & Therapie \\
\hline Netdoktor.de & Entstehung & Symptome & $\mathrm{M}=2,05$ \\
\hline User-Urteil & $\mathrm{M}=2,03$ & $\mathrm{M}=1,90$ & $\mathrm{M}=0,22$ \\
Experten-Urteil & $\mathrm{M}=0,23$ & $\mathrm{M}=0,00$ &
\end{tabular}

\section{Fazit und Diskussion}

Drei Qualitätsdimensionen, nämlich Inhaltsqualität (z. B. Vollständigkeit und Richtigkeit der Darstellung), Darstellungsqualität (z. B. Navigation und Design) sowie technische Qualität (z. B. Ladezeiten und Sicherheit) wurden als bedeutsam zur Beurteilung von Gesundheitskommunikation im Internet und insbesondere von Webseiten aus dem Bereich Health Content herausgestellt. In einer empirischen Studie wurden dazu 101 User im Labor zu den Gesundheitswebseiten Lifeline.de, Gesmed.ch und Netdoktor.de nach ihren allgemeinen und auf konkreten Suchaufträgen zu den Krankheiten Bandscheibenvorfall, Diabetes oder Depression beruhenden Urteilen zur Inhalts-, Darstellungs- und technischen Qualität befragt.

Es zeigte sich, dass die befragten User sehr differenzierte Qualitätsurteile abgaben. Werden konkrete Suchaufträge und Probleme mit Hilfe der Webseiten zu lösen versucht, so werden auf einer bestimmten Webseite die spezifischen Kriterien der Inhaltsqualität wie Vollständigkeit, Verlässlichkeit und Einfachheit unabhängig voneinander beurteilt. Die Ergebnisse weisen darauf hin, dass die in der Studie gewählten Qualitätskriterien unterscheidbar sind, und darauf, dass die User keine anfänglichen Pauschalurteile im Sinne eines Halo-Effektes fällen (z. B. aufgrund des Designs einer Webseite) und in Abhängigkeit davon alle folgenden Qualitätsurteile generalisieren.

Dieser Eindruck wird auch bestätigt, wenn man die allgemeine Bewertung einer Webseite anhand eines ersten Eindrucks mit den Bewertungen nach der Ausführung eines konkreten Suchauftrages vergleicht. Die allgemeine Bewertung ist zunächst recht unspezifisch, und zwischen verschiedenen Webseiten wird kaum unterschieden. Es ist also zu vermuten, dass ein erster Eindruck weniger an inhaltlichen Kriterien orientiert ist. Erst bei der problemorientierten Nutzung werden differenziertere Urteile gefällt. Erstens bestehen signifikante Unterschiede zwischen den Bewertungen, die aufgrund einer unspezifischen Nutzung und einer problemspezifischen Suche gefällt werden. Zweitens sind nach der problemorientierten Suche große Differenzen in der Bewertung der untersuchten Webseiten festzustellen. Auffällig ist also, dass der zunächst zugestandene Vertrauensbonus je nach Qualität der Seite entweder entzogen (die Seite wird schlechter bewertet) oder erweitert wird (die Seite wird besser bewertet als am Anfang). Das spricht für einen medienkompetenten Umgang der User, die sich nicht nur von einem ersten intuitiven Eindruck leiten lassen. 
Besondere Bedeutung - sowohl für Anbieter von Gesundheitswebseiten als auch für User - hat die Gewichtung der Qualitätsdimensionen und welche der Qualitätskriterien letztlich für die Weiternutzung der Seite ausschlaggebend sein könnten. Die Gesamtbewertung einer gesundheitsbezogenen Webseite und ob man in Erwägung zieht, diese weiter zu nutzen, ist von Webseite zu Webseite von verschiedenen Faktoren abhängig. Als wichtige Prädiktoren können die als korrekt empfundene Darstellung der Krankheitsentstehung, die wahrgenommene Vollständigkeit der dargestellten Inhalte und die übersichtliche Navigation auf der Webseite gelten. Ebenso wie in vorhergehenden Studien wurde also die Navigation als besonders wichtiges Kriterium für die Gesamtbeurteilung herausgestellt, weniger ließen sich die Befragten unserer Studie vom Design, dem Layout und der Aufmachung einer Seite leiten (vgl. Eysenbach \& Köhler, 2002). Dies ist möglicherweise darauf zurückzuführen, dass sie tatsächlich gezwungen waren, die inhaltliche Qualität anhand von konkreten Suchaufträgen zu bewerten. Damit erscheinen die User insgesamt sehr medienkompetent und qualifiziert, die vorhandenen Angebote auch unabhängig von stilistischen Aspekten zu bewerten und zu vergleichen.

In ihrer Qualitätsbewertung kommen User und Experten zu weitgehend ähnlichen Urteilen. Offensichtlich können User die Qualität recht gut einschätzen. Dieses Ergebnis ist besonders überzeugend, weil auch die Übereinstimmung der Expertenurteile vergleichsweise hoch ist (vgl. Craigie et al., 2002). Damit ist die vorliegende Studie die einzige im deutschsprachigen Raum, die User- und Expertenurteile bei der Bewertung von Gesundheitskommunikation im Internet erfasst und deren Qualitätsurteile vergleicht. Dennoch können die Ergebnisse zunächst nur als erste Tendenzen interpretiert werden. Denn aufgrund der Tatsache, dass User und Experten zu ähnlichen Evaluationsergebnissen kommen, ist natürlich nicht alle „Gefahr“ der Gesundheitskommunikation über das Internet gebannt. Wir können annehmen, dass die Laien ein gutes Gespür für inhaltliche Qualität haben, jedoch im Einzelfall nicht davon ausgehen, dass die User fachlich immer ausreichend kompetent sind, richtige von falschen Informationen zu unterscheiden, die Informationen auf medizinische Angemessenheit und Vollständigkeit zu prüfen und angemessen damit umzugehen.

Darüber hinaus ist die Stichprobe der User nicht repräsentativ, und es wurden Personen befragt, die nicht an den Krankheiten leiden, zu denen sie Suchaufträge bearbeitet und anhand derer sie die Webseiten bewertet haben. Damit ist ihre Suchmotivation vermutlich wesentlich weniger involviert und handlungsrelevant im Vergleich zu Usern, die selbst von einer Krankheit betroffen sind und deshalb die entsprechenden Informationen benötigen. Gleichzeitig könnten die in der Studie befragten User besonders kritisch vorgegangen sein, da sie im Labor zu Aspekten der Qualität befragt wurden und die Fragen damit einen Hinweischarakter erhielten. Auch andere Arten von Laboreffekten sind vorstellbar. Vermutlich recherchieren User im Labor, die einen konkreten Fragenkatalog abarbeiten, wesentlich stringenter als Betroffene im Feld. Auch die zeitliche Begrenzung einer Laborstudie ist nicht mit einer echten Nutzungssituation vergleichbar. In nachfolgenden Studien zu diesem Thema sollte möglichst mit repräsentativen Stichproben gearbeitet werden, um die Bedeutung der Moderatorvariablen wie der eigenen Betroffenheit klären zu können. Auch ist die Erweiterung der Stichprobe der Experten, sowohl was ihre Anzahl als auch die Erfahrungshintergründe anbelangt, für weitere Studien ratsam. Eine repräsentative Expertenstichprobe wird jedoch kaum realisierbar sein, da der Expertenstatus vorab definiert werden muss. Können nur Schulmediziner als echte Experten gelten, oder alle Personen, die sich professionell mit einem medizinischen Thema befassen, oder sind nur Wissenschaftler echte Experten? Weil die- 
se Definition nie allgemein gültig sein kann, wird jede Studie zur Qualitätsbeurteilung subjektiv-normativen Charakter behalten und muss vor dem Hintergrund der Stichprobenziehung und der (subjektiv erstellten) Kriterienkataloge interpretiert werden.

\section{Literatur}

Ambre, J., Guard, R., Perveiler, F. M., Renner, J. \& Rippen, H. (1997). Criteria for assessing the quality of health information on the Internet. Agency for Health Care Policy and Research [Online]. Erhältlich: http://hitiweb.mitretek.org/docs/criteria.html [2004, 28. Juli].

American Psychiatric Association (2003). Diagnostisches und statistisches Manual psychischer Störungen: Textrevision; DSM-IV-TR (übersetzt nach der Textrevision der 4. Auflage des Diagnostic and statistical manual of mental disorders der American Psychiatric Association). Göttingen: Hogrefe.

Anderson, G. B. J. (1991). The Epidemiology of Spinal Disorder. In J. W. Frymoyer (Ed.), The Adult Spine: Principles and Practice. New York: Raven Press.

Barnes, M. D., Penrod, C., Neiger, B. L., Merrill, R. M., Thackeray, R., Eggett, D. L. \& Thomas, E. (2003). Measuring the Relevance of Evaluation Criteria among Health Information Seekers on the Internet. Journal of Health Psychology, 8 (1), 71-82.

Beck, K., Schweiger, W. \& Wirth, W. (Hrsg.) (2004). Gute Seiten - schlechte Seiten. Qualität in der Onlinekommunikation. München: Reinhard Fischer.

Bowen, D. J., Ludwig, A., Bush, N., Unruh, H. K., Meischke, H., Wooldridge, J. A. \& Robbins, R. (2003). Early Experience with a Web-based Intervention to Inform Risk of Breast Cancer. Journal of Health Psychology, 8 (1), 175-186.

BSMO (2003). Nutzung und Nutzer von Gesundheitsinformationen im Internet [Online]. Erhältlich: http://www.bsmo.de/misc/Prsentation_030623_afgis_jh.pdf [2004, 12. Juli].

Commission of the European Communities (2002). eEurope 2002: Quality Criteria for Health related Websites. Commission of the European Communities [Online]. Erhältlich: http://euro pa.eu.int/information_society/eeurope/ehealth/doc/communication_acte_en_fin.pdf [2004, 28. Juli].

Craigie, M., Loader, B., Burrows, R. \& Muncer, S. (2002). Reliability of health information on the Internet: an examination of experts' ratings. Journal of Medical Internet Research 4 (1): e2 [Online]. Erhältlich: http://jmir.org/2002/1/e2/index.htm [2004, 23. Februar].

Cramer, H. \& Stiehler, W. (2000). Virtueller Arztbesuch. Medizinische Beratung im Web: Kommt das E-Health-Business? In c't, 9/2000 v. 25.04.00: 102-107.

Dahinden, U., Kaminski, P. \& Niederreuther, R. (2004). Gemeinsamkeiten und Unterschiede bei der Qualitätsbeurteilung aus Angebots- und Rezipientenperspektive. In K. Beck, W. Schweiger \& W. Wirth (Hrsg.), Gute Seiten - schlechte Seiten. Qualität in der Onlinekommunikation (S. 103-126). München: Reinhard Fischer.

Dutta-Bergman, M. J. (2003). Trusted Online Sources of Health Information: Differences in Demographics, Health Beliefs, and Health-Information Orientation. Journal of Medical Internet Research, 5 (3): e21 [Online]. Erhältlich: http://jmir.org/2003/3/e21/index.htm [2004, 23. Februar].

Dutta-Bergman, M. J. (2004). The Impact of Completeness and Web Use Motivation on the Credibility of e-Health Information. Journal of Communication, 54 (2), 253-269.

Emnid (2003). Onliner ziehen Internet in Gesundheitsfragen zu Rate TNS EMNID befragt Internet-Nutzer zu Arztbesuch und Internet [Online]. Erhältlich: http://www.agz-rnk.de/agz/content/2/aktuelles/akt_00028.php [2004, 03. August].

Eysenbach, G. \& Diepgen, T. L. (1998). Towards quality management of medical information on the internet: evaluation, labelling, and filtering of information. British Medical Journal, 317, 1496-1500.

Eysenbach, G. \& Köhler, C. (2002). How do consumers search for and appraise health information on the world wide web? Qualitative study using focus groups, usability tests, and in-depth interviews. British Medical Journal, 324, 573-577. 
Eysenbach, G., Powell, J., Kuss, O., Sa, E.-R. (2002). Empirical studies assessing the quality of health information for consumers on the world wide web. A systematic review. Journal of the American Medical Association, 287 (20), 2691-270.

Fox, S., Rainie, L., Horrigan, J., Lenhart, A., Spooner, T., Burke, M., Lewis, O. \& Carter, C. (2000). The Online Health Care Revolution: How the Web helps Americans Take Better Care of themselves. Pew Internet \& American Life Project: Online life report [Online]. Erhältlich: http://www.pewinternet.org [2004, 28. Juli].

Fox, S. \& Fallows, D. (2003). Internet Health Resources. Pew Internet E American Life Project [Online]. Erhältlich: http://www.pewinternet.org [2004, 28. Juli].

Früh, W. (2001). Inhaltsanalyse. Theorie und Praxis (5. überarb. Auflage ed.). München: Ölschläger.

Griffiths, K. M. \& Christensen, H. (2000). Quality of web based information on treatment of depression: cross sectional survey. British Medical Journal, 321, 1511-1515.

Hägele, M. \& Leopold, C. (2004). www.medinfo.de macht wichtige Qualitätsauszeichnungen medizinischer Webseiten erstmals gesammelt sichtbar. In A. Jäckel (Hrsg.), Telemedizinführer Deutschland, Ausgabe 2004 (S. 7-10). Ober-Mörlen: Deutsches Medizinforum.

Harris Interactive (2001). The Increasing Impact of eHealth on Consumer Behavior. Harris Interactive Health Care News, 1 (21). [Online]. Erhältlich: http://www.harrisinteractive.com/ news/newsletters_healthcare.asp [2004, 28. Juli].

Hautzinger, N. (2003). Pharmakommunikation im Internetzeitalter. Theorie und Praxis eines patientenorientierten Kommunikationsmanagements am Beispiel der Pharmabranche Schweiz. München: Reinhard Fischer.

Hautzinger, N. (2004). Health Content im Internet - Aspekte der Qualitätssicherung. In K. Beck, W. Schweiger \& W. Wirth (Hrsg.), Gute Seiten - schlechte Seiten. Qualität in der Online-Kommunikation. München: Reinhard Fischer.

Health On the Net Foundation (2002). HON-Surveys [Online]. Erhältlich: http://www.hon.ch/ Survey/analysis.html [2004, 28. Juli].

Herbenstreit, S. \& Güntert, B. (2001). Qualitätsaspekte der Online-Gesundheitskommunikation. In K. Hurrelmann \& A. Leppin (Hrsg.), Moderne Gesundheitskommunikation. Vom Aufklärungsgespräch zur E-Health (S. 277-289). Bern u. a.: Huber.

Herold, G. (2004). Innere Medizin 2004. Köln: Selbstverlag.

Horrigan, J. B. \& Rainie, L. (2002). Getting Serious Online. Pew Internet E American Life Project [Online]. Erhältlich: http://www.pewinternet.org [2004, 28. Juli].

Houston, T. K. \& Allison, J. J. (2002). Users of Internet Health Information: Differences by Health Status. Journal of Medical Internet Research, 4 (2): e7 [Online]. Erhältlich: http://jmir.org/ 2002/2/e7/index.htm [2004, 23. Februar].

Identity Foundation (2001). Gesundheitsstudie: Die Deutschen und ihre Einstellungen zu Krankheit und Gesundheit. Identity Foundation [Online]. Erhältlich: http://www.identityfoundati on.de/was_wir_tun.htm [2004, 1. Juni].

Impicciatore, P., Pandolfini, C., Casella, N. \& Bonati, M. (1997). Reliability of health information for the public on the world wide web: systematic survey of advice on managing fever in children at home. British Medical Journal, 314, 1875.

International Organization for Standardization (1994). Technical Committee ISO/TC 176. ISO 8402: Quality management and quality assurance. Vocabulary (2nd ed). Geneva: International Organization for Standardization.

Kim, P., Eng, T. R., Deering, M. J. \& Maxfield, A. (1999). Published criteria for evaluating health related web sites: review. British Medical Journal, 318, 647-649.

Manfredi, M. P. \& Covington, T. R. (2000). The Role of Self-Care and Nonprescription Drug Therapy in Managing Illness: The Rx-to-OTC Switch Movement. In D. Nash, M. P. Manfredi, B. Bozarth \& S. Howell (Hrsg.), Connecting with the new Healthcare Consumer: Defining your Strategy (S. 237-264). New York u. a.: McGraw Hill.

Media Perspektiven (2003). Basisdaten. Daten zur Mediensituation in Deutschland 2003. Frankfurt: Eigenverlag. 
Neuberger, C. (2004). Qualität im Online-Journalismus. In K. Beck, W. Schweiger \& W. Wirth (Hrsg.), Gute Seiten - schlechte Seiten. Qualität in der Onlinekommunikation (S. 32-57). München: Reinhard Fischer.

Neuhauser, L. \& Kreps, G. L. (2003a). The Advent of E-Health. How Interactive Media Are Transforming Health Communication. Medien E Kommunikationswissenschaft, 51 (3/4), 541-557.

Neuhauser, L. \& Kreps, G. L. (2003b). Rethinking Communication in the E-health Era. Journal of Health Psychology, 8 (1), 7-23.

o. V. (2003). Glückssache: Medizinische Beratung im Internet. test, 4, 88-91.

Pandolfini, C. \& Bonati, M. (2002). Follow-up of quality of public oriented health information on the world wide web: Systematic re-evaluation. British Medical Journal, 324, 582-583.

Quandt, T. (2004). Qualität als Konstrukt. Entwicklung von Qualitätskriterien im Onlinejournalismus. In K. Beck, W. Schweiger \& W. Wirth (Hrsg.), Gute Seiten - schlechte Seiten. Qualität in der Onlinekommunikation (S. 58-79). München: Reinhard Fischer.

Reiß, B. (2000). Das Internet als Quelle medizinischer Information. Unveröffentlichte Magisterarbeit, Universität Münster.

Rimal, R. N. \& Adkins, A. D. (2003). Using Computers to Narrowcast Health Messages: The Role of Audience Segmentation, Targeting and Tailoring in Health Promotion. In T. L. Thompson, A. M. Dorsey, K. I. Miller \& R. Parrott (Hrsg.), Handbook of Health Communication (S. 497-513). Mahwah, N. J.: Lawrence Erlbaum.

Schatz, H. \& Schulz, W. (1992). Qualität von Fernsehprogrammen. Kriterien und Methoden zur Beurteilung von Programmqualität im dualen Fernsehsystem. Media Perspektiven, o. Jg. (11), 690-712.

Schmidt-Kaehler, S. (2003). Internetkompetenz für Menschen mit chronischen Erkrankungen. Veröffentlichungsreihe des Instituts für Pflegewissenschaft an der Universität Bielefeld (IPW). Bielefeld: Universität Bielefeld.

T-Online (2002). Kurzstudie T-Online Panel 9/2002. Käufer rezeptfreier Gesundheitspräparate Surfverhalten im Internet. T-Online [Online]. Erhältlich: http://www.t-online.de/werbung [2004, 28. Juli].

Vlasic, A. (2004). Über Geschmack lässt sich nicht streiten - über Qualität schon? Zum Problem der Definition von Maßstäben für publizistische Qualität. In K. Beck, W. Schweiger \& W. Wirth (Hrsg.), Gute Seiten-schlechte Seiten. Qualität in der Onlinekommunikation (S. 15-31). München: Reinhard Fischer. 